\title{
LA GESTIÓN DEL CONOCIMIENTO COMO DISCIPLINA EMPRESARIAL
}

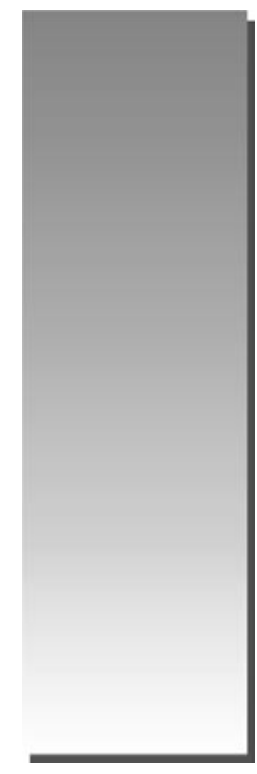

Juan Puell Palacios *

E-mail: juanpuell@hotmail.com

\begin{abstract}
RESUMEN
La Gestión del Conocimiento es un nuevo paradigma organizacional que las empresas actuales tienen que considerar no solamente para ser más competitivas en los mercados locales o internacionales, sino para sobrevivir creando nuevos productos y servicios. El conocimiento es un constructo mental que cae en lo abstracto y, por lo tanto, no se puede gestionar; lo que realmente se gestionan son los activos del conocimiento.
\end{abstract}

Existen varios tipos de conocimiento como por ejemplo al conocimiento tácito, el conocimiento implícito y el conocimiento explicito; la pregunta es ¿cuál de estos conocimientos se va a gestionar? Consideramos que se deben gestionar los conocimientos que tienen un nivel de veracidad, que tienen posibilidad de aplicación práctica y aquellos que son nuevos porque, debido a que no existen en ninguna otra parte, se convierten en algo que puede constituir en un invento o un descubrimiento que contribuya a satisfacer una necesidad o un deseo, a mejorar la calidad de vida de los seres vivos, o a enriquecer un campo teórico científico; al mismo tiempo es un conocimiento que se puede convertir en un producto o servicio.

El conocimiento es propio de las personas y no de las organizaciones que son entelequias jurídicas que no piensan. El conocimiento implícitamente es personal pero también puede ser colectivo o grupal; cuando tiene esta característica es participativo, es decir, es producto de una discusión o debate y es este producto el que tiene que difundirse en las organizaciones para lograr su aceptación y comprensión.

La innovación de los productos también constituye un nuevo conocimiento. Los productos o servicios no siempre tienen las mismas características o bondades, ya que éstas pueden cambiar en la forma o en el fondo, pero con la condición de que el usuario o cliente sienta complacencia y satisfacción con esta modificación. Debemos tener amor al conocimiento porque lo importante no es solamente poseerlo sino aspirar a conocer más; recordemos que los conocimientos profundos colindan con la sabiduría.

* Estudios de Doctorado en la Universidad Nacional Federico Villarreal y de Maestría en la Universidad Nacional Mayor de San Marcos. Licenciado en Administración. Director de la Escuela Académico Profesional de Administración de la Facultad de Ciencias Administrativas (UNMSM). Docente de la Universidad Ricardo Palma. 
El conocimiento sirve para entender mejor, para comprender e interpretar lo que nos rodea externamente y también lo que nos llena internamente. Nos permite analizar el entorno empresarial con todos sus factores y el mundo interno de la organización.

La gestión del conocimiento comprende objetos de difícil análisis como la persona, su mente, su voluntad de participación social. El talento personal es la capacidad particular de un individuo para alcanzar resultados en una determinada empresa asumiendo un compromiso y una acción en ciertas circunstancias.

Las organizaciones deben desarrollar programas continuos de capacitación y desarrollo de los empleados; sino se implementan estos programas no se puede llegar a conocer la excelencia de un gran cuerpo de conocimientos, aunque esté entre nosotros. Recibir nuevos conocimientos es estimulante, motivacional e indispensable para un hombre de negocios.

Palabras clave: Gestión del conocimiento, conocimiento, el conocimiento al interior de las organizaciones.

\section{ABSTRACT}

Knowledge Management is a new organizational paradigm that companies, today, have to consider not just to be more competitive in local markets or international, but to survive creating new products and services. Knowledge is a mental construct that falls on the abstract and, therefore, can not manage, what they really are managed are knowledge assets.

There are several kinds of knowledge such as the tacit knowledge, implicit and explicit knowledge, the question is which of these skills are going to manage? We believe that knowledge must be managed to have a level of accuracy, that have the possibility of practical application and those who are new, because they do not exist anywhere else, become something that can constitute an invention or discovery that contributes to satisfy a need or desire, to improve the quality of life of human beings, or to enrich a field theoretical scientist at the same time is knowledge that can be converted into a product or service.

Knowledge is typical of the people, not organizations that are not legal Entelequia think. The implicit knowledge is personal but can also be collective or group; when you have this feature is participatory, further, is the product of a discussion or debate, and this product is the one that has to be disseminated in organizations to achieve their acceptance and understanding.

Product innovation is also a new knowledge. The products or services do not always have the same characteristics or virtues, as they may change in form or substance, but on condition that the user or customer feels pleasure and satisfaction with this change. We must have love for knowledge because it is not just possess it, but hope to learn more, remember that knowledge adjacent to the profound wisdom.

The knowledge used to better understand, to understand and interpret what surrounds us externally and also what fills us internally. It allows us to analyze the business environment with all its factors and the inner world of the organization.

Knowledge management comprises objects of analysis difficult as the person, his mind, his desire for social participation. The talented staff is the ability of a particular individual to achieve results in a company making a commitment and action in certain circumstances.

Organizations must develop ongoing training and development of employees, these programs are implemented but you can not get to know the excellence of a large body of knowledge, even if it is between us. Receive new knowledge is inspiring, motivational and indispensable for a businessman.

Key words: Knowledge Management, knowledge, the internal knowledge of the organizations. 
La gestión del conocimiento es una nueva disciplina que está interesando más a los empresarios porque permite que las organizaciones tengan una performance cada vez más inteligente. Esta disciplina la entendemos como un conjunto de principios, métodos, técnicas, herramientas, matrices y tecnologías que permiten obtener los conocimientos precisos y adecuados en el tiempo oportuno, de la forma más eficiente y sencilla, con el fin de conseguir una actuación institucional lo más inteligente posible.

El conocimiento en sí mismo es algo abstracto para que se pueda gestionar; lo que se gestiona en una organización son los recursos que se emplean en ella, optimizándolos y haciéndolos más productivos y sostenibles. Sin embargo lo que se puede gestionar del conocimiento son sus activos para crear y desarrollar más y nuevos conocimientos.

Son activos del conocimiento las bases de datos y los conocimientos de cada persona tales como experiencia, capacidades, habilidades competitivas y todo tipo de conocimientos asimilados o aprendidos, ya sea tecnológicos o de cualquier otra manifestación.

\section{CONOCIMIENTO Y ORGANIZACIONES}

Para gestionar los activos del conocimiento es bueno tener una idea suficientemente clara sobre este concepto. Platón decía que el conocimiento puede definirse como "una creencia cierta justificada"; en otras palabras, para decir que poseemos un conocimiento debemos albergar una creencia; esta creencia debe ser objetivamente verdadera y su veracidad se debe sustentar en hechos suficientemente sólidos.

Los que laboramos en las organizaciones debemos saber concienzudamente qué entendemos por conocimiento. Existen varios conceptos o definiciones de conocimiento, así por ejemplo Turan (1932) nos dice que es la “... información que ha sido organizada para hacerla entendible $y$ aplicable a la solución de problemas o a la toma de decisiones".

El académico Agusti Canals considera que:

"Conocimiento es el conjunto de expectativas que tiene un agente, la distribución de probabilidades que él asigna a los posibles sucesos que pueden pasar en su entorno. Es decir, la visión que él tiene de cómo son las cosas y cómo se van a comportar."
En las organizaciones empresariales se presentan algunas situaciones que requieren una profunda reflexión y análisis de su estructura, como por ejemplo, los niveles de conocimiento que deben existir al interior de ellas, que son el conocimiento de la propia organización y el conocimiento de las personas que la conforman.

Las organizaciones sociales son incomprensibles sin la presencia de las personas, sin las cuales el conocimiento seria un espacio vacío e infinito. Son las personas las únicas que generan conocimiento y para ello deben tener en cuenta los avances tecnológicos y el contexto globalizado. El empresario moderno, actualizado y con visión de futuro, está obligado a desarrollar programas tendientes a formar y/o capacitar a los técnicos, especialistas, expertos y profesionales en general, para lograr la creación de nuevos conocimientos y su transmisión inmediata o materialización práctica.

Las organizaciones son sistemas complejos que pueden dar interesantes indicios para lidiar con problemas prácticos que difícilmente podremos resolverlos con una mentalidad lineal y mecanicista. La misma complejidad de la propia organización permite generar conocimientos que aún no son difundidos en ninguna parte.

Para el filósofo austriaco Karl Popper todo conocimiento es provisional. Una simple suposición puede ser utilizada y considerada como conocimiento mientras no aparezca un hecho que la contradiga; en ese momento dejaría de ser conocimiento, por ello el ser humano, muchas veces, tiene que desaprender lo aprendido

El conocimiento de la organización relacionado con su visión del entorno reside básicamente en tres cuestiones:

- Las personas. Cada persona dentro de la organización posee su propio conocimiento que lo utiliza para orientar sus acciones, pero a la vez también tiene sus propios objetivos individuales que no necesariamente son compatibles con los de la organización.

- Las tecnologías de la información. La información puede convertirse en conocimiento útil cuando a la vez tiene una aplicación en la praxis. La información puede representarse en forma de conocimiento cuando representa un dato importante.

- Los procesos. Para ser reformulados o cambiados totalmente requieren conocimientos. Un 
nuevo proceso de producción o administrativo encierra conocimientos novedosos porque es diferente y no existe en ninguna otra parte. Si tuviera un error se puede corregir y, si no se corrige, conllevará a que se cometa otro error.

\section{CÓMO GESTIONAR EL CONOCIMIENTO}

Hace una década nadie se pronunciaba acerca de la gestión del conocimiento, pero en el mundo empresarial este concepto cada vez requiere mayor atención, porque el conocimiento es uno de los motivos más poderosos que permitirá a la empresa sobrevivir, crecer y desarrollarse.

La gestión del conocimiento obedece a tres tendencias sociales y económicas: la globalización, la ubicuidad de las tecnologías de la información (TIC) y la visión.

- La globalización. Ha permitido que las empresas se deslocalicen y ofrezcan sus productos en mercados más amplios, además de generarse mayores niveles de competencia. Para competir en nuevos mercados se requiere saber qué sabemos y qué deberíamos saber.

- La ubicuidad de las tecnologías de la información. La cantidad de información a la que se tiene acceso hoy en día es abundante y espectacular; sin embargo la productividad en el trabajo no ha crecido, específicamente en el lado humano. Precisamente es esa productividad tan baja la que ha permitido que los gerentes de las empresas se vean obligados a resaltar y promover la importancia y el valor de las capacidades, en términos de conocimiento que deben tener las personas dentro de la organización para poder utilizar esa abundante información.

- La visión. Generalmente se centra en el conocimiento tácito de las organizaciones más el conocimiento explícito de las mismas. La visión es el reflejo de las capacidades de las personas que puede tener ciertas limitaciones en su implementación y efectividad.

Para generar nuevos conocimientos los autores Scott Cook y John Seeley Bros utilizan la separación entre conocimiento tácito y explícito, entre conocimiento individual y grupal, para poder definir cuatro formas de conocimiento que corresponden a las combinaciones de esas categorías. De acuerdo a lo manifestado por estos autores existiría un conocimiento individual explícito que se llama conceptos; un conocimiento grupal explícito que son las historias; otro conocimiento individual tácito que son las habilidades, y un conocimiento grupal tácito que se denomina género. Los autores también proponen la introducción de un nuevo concepto complementario al de conocimiento que se llama "conocer". La diferencia consiste en que, mientras que el conocimiento es algo que se posee, algo estático, el conocer es algo que se produce en el momento de la acción, es algo dinámico. Es ese conocimiento el que permite, a través de la acción, la utilización de las diversas formas de conocimiento para realizar actividades concretas y, al mismo tiempo, generar nuevo conocimiento.

Se produce una complementariedad entre lo que los autores llaman la epistemología de la posesión, la del conocimiento, y la epistemología de la práctica, la del conocer. A esto podemos decir que la suma de conocer y conocimiento permite entender la relación entre lo que conocemos y lo que hacemos.

\section{INVERTIR EN CONOCIMIENTO PARA GESTIONAR CONOCIMIENTO}

Algunas empresas utilizan sistemas de incentivos para conseguir que el conocimiento sea compartido, no solamente dentro de la organización sino también fuera de ella. Para ello utilizan una serie de estrategias para fomentar la "compartición” del conocimiento (ver cuadro $\mathrm{N}^{\circ}{ }^{\circ}$ ).

El conocimiento debe ser compartido, estamos de acuerdo, pero ¿hasta dónde se puede compartir el conocimiento?, ¿con quién se debe compartir?, ¿quién decide si se debe o no compartir? Se debe tener en cuenta que los servicios de inteligencia de los estados y de las empresas dedican gran cantidad de recursos a la protección del conocimiento propio o al intento de apropiación del conocimiento ajeno. Las últimas técnicas de espionaje industrial que se aprovechan de las redes del área local inalámbricas son una prueba contundente de ello.

Los gerentes de las organizaciones, los estados y los individuos tienen importantes incentivos $\mathrm{y}$ motivos que hacen que sean muy reservados para compartir conocimiento. La gestión del 
Cuadro N. ${ }^{\circ}$ 1. Estrategias para comparticionar el conocimiento.

\begin{tabular}{|c|c|c|c|}
\hline \multicolumn{4}{|c|}{ ESTRATEGIAS } \\
\hline $\begin{array}{l}\text { Selección de empleados } \\
\text { proclives a compartir }\end{array}$ & $\begin{array}{l}\text { Desarrollar un clima } \\
\text { de confianza }\end{array}$ & Crear comunidades & $\begin{array}{l}\text { Fomentar la com- } \\
\text { partición como } \\
\text { valor central }\end{array}$ \\
\hline $\begin{array}{ll}- & \text { Buena selección de per- } \\
\text { sonal. } \\
\text { - } & \text { Trabajo en equipo. } \\
\text { - } & \text { Compartir experiencias. } \\
\text { - } & \text { Involucrar a los em- } \\
\text { pleados que están en el } \\
\text { proceso. }\end{array}$ & $\begin{array}{l}\text { - Crear una atmósfera } \\
\text { de confianza. } \\
\text { - Crear un código } \\
\text { ético. } \\
\text { - Fomentar una } \\
\text { cultura del cono- } \\
\text { cimiento. }\end{array}$ & $\begin{array}{ll} & \text { Crear grupos con in- } \\
\text { tereses comunes. } \\
\text { - } \\
\text { Crear comunidades } \\
\text { virtuales en Internet. }\end{array}$ & $\begin{array}{l}\text { Subrayar la im- } \\
\text { portancia de } \\
\text { compartir el } \\
\text { conocimiento. } \\
\text { Ánimo para com- } \\
\text { partir el cono- } \\
\text { cimiento. }\end{array}$ \\
\hline $\begin{array}{l}\text { Programas de compar- } \\
\text { tición de conocimiento }\end{array}$ & $\begin{array}{c}\text { Reorganizar } \\
\text { adecuadamente }\end{array}$ & Crear comunidades & $\begin{array}{l}\text { Fomentar la apari- } \\
\text { ción de líderes }\end{array}$ \\
\hline $\begin{array}{l}\text { Apoyo de la Alta Direc- } \\
\text { ción. } \\
\text { - } \\
\text { Programas de moti- } \\
\text { vación para compartir. }\end{array}$ & $\begin{array}{ll}\text { - } & \text { El empleado puede } \\
\text { pertenecer a varios } \\
\text { grupos. } \\
\text { - } \\
\text { Compartir en equi- } \\
\text { pos. }\end{array}$ & $\begin{array}{l}\text { - } \text { Crear grupos con in- } \\
\text { tereses comunes. } \\
\text { - } \text { Crear comunidades } \\
\text { virtuales en Internet. }\end{array}$ & $\begin{array}{l}\text { Buscar líderes } \\
\text { que arrastren } \\
\text { a toda la orga- } \\
\text { nización. }\end{array}$ \\
\hline
\end{tabular}

conocimiento requiere del apoyo directo de la Alta Dirección para lograr el éxito empresarial; esto implica reconocer que el recurso más escaso ya no es el capital sino el conocimiento que poseen las empresas. Chris Bartlett, profesor de la Universidad de Harvard, sostiene que las bases organizativas que funcionaban bien en la economía industrial ya no sirven para la economía del conocimiento, y concluye que las claves de la nueva organización son tres:

- Un concepto de la organización basado en las personas y en las relaciones entre ellas, más que en los procedimientos y las funciones.

- Un cambio en el rol de los directivos ya que más que estrategas, controladores, e implementadores, deben ser líderes capaces de construir y desarrollar la organización, así como emprendedores capaces de innovar.

- Una nueva filosofía de gestión basada en los objetivos, los procesos y las personas. Esta nueva filosofía debe ser la filosofía del éxito, el mismo que depende de la preparación. Sin preparación habrá siempre fracaso; cuando se determinan estratégicamente los principios generales no habrá confusión en la implementación de los planes estratégicos.

\section{LINEAMIENTOS PARA LA GESTIÓN DEL CONOCIMIENTO}

Agusti Canals, un experto en Gestión del Conocimiento, propone diez lineamientos para gestionar el conocimiento que, desde mi punto de vista, son fundamentales en la gestión gerencial que busca éxito en un mercado globalizado:

1. Nuevas tecnologías para la gestión de contenidos, con especial atención a las que permitan gestionar los formatos audiovisuales.

2. Explorar la relación conocimiento tácitoconocimiento explícito, no como una oposición sino como una complementariedad.

3. Profundizar en una distinción clara entre datos, información y conocimiento que permita identificar los que realmente son contenidos cognitivos desde un punto de vista práctico, e implementar las metodologías adecuadas.

4. Conocer más a fondo el fenómeno del conocimiento colectivo y sus mecanismos.

5. El conocimiento como fenómeno emergente que se genera en las organizaciones, entendidas como sistemas complejos adaptativos.

6. Tecnologías que ayudan a la comunicación y a la interrelación en organizaciones en red. 
7. Introducción de la variable espacio-temporal: ¿en qué cosas, si es que las hay, el conocimiento sólo puede transmitirse de manera presencial y síncrona?

8. La relación entre la gestión del conocimiento y el e-learning.

9. Continuar el desarrollo y aplicación de la teoría de la empresa basada en el conocimiento.

10.Diseñar sistemas para determinar el retorno de la inversión (RDI) en proyectos de gestión del conocimiento y para medir el capital intelectual.

Desde mi punto de vista los lineamientos propuestos por Agusti Canals son muy interesantes, pero pueden haber muchos más como por ejemplo la capacitación continua para generar nuevos conocimientos, la conformación de talleres de investigación y desarrollo, etc.

\section{BIBLIOGRAFÍA}

Autores varios (2005) Gestión del Conocimiento. Harvard Business Review.

Canals, Agusti (2003). Gestión del Conocimiento. Editorial Gestión 2000.

Del Moral, Anselmo; Pazos, Juan; Rodríguez, Esteban; Rodríguez - Patrón, Alonso y Suárez, Sonia (2007). Gestión del Conocimiento. International Thomson Editores Spain Paraninfo S. A.

Edvisson, Leif y Malone, Michael S. (2004) El Capital Intelectual. Editorial Norma. 